\title{
Les Chroniques
}

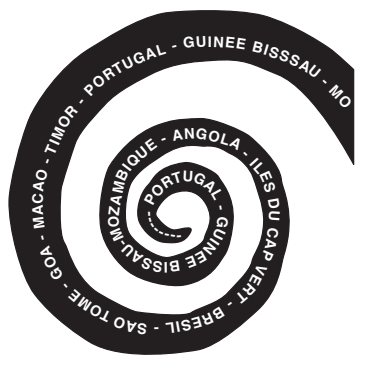




\section{PERSISTANGE DU LUSOTROPIGALISME AU XXI ${ }^{\mathrm{e}}$ SIÈGLE**}

Il n'est jamais facile de commenter un dictionnaire. Quand il s'agit d'un dictionnaire de vocation encyclopédique, la difficulté s'accroît, car il faut pouvoir procéder non seulement à la critique de la conception d'ensemble, mais aussi du plus grand nombre possible d'entrées. Le «Dictionnaire thématique de la lusophonie » est un dictionnaire encyclopédique, mais il n'est pas construit comme une encyclopédie de thèmes sur chacun des espaces concernés. En principe, il s'agit de ceux liés à la «lusophonie » en tant que telle, mais les auteurs n'ont pas poussé ce choix jusqu'au bout puisqu'on a des notices sur des territoires qui ne devraient pas (ou plus) être considérés comme lusophones: si la Galice du Royaume d'Espagne y a justement sa place, pourquoi en revanche y trouve-t-on la Casamance, Goa et Macao? Ou alors il fallait borner historiquement ou socialement le propos: personne ne parle plus portugais à Goa, et dans l'espace casamançais, le lusocréole concerne un milieu social bien particulier et en aucun cas la population de la région. Quitte à intégrer ces espaces, il aurait alors fallu les placer dans le chapitre Património ou História et non dans celui des Regiões lusófonas. La «lusophonité » de ces territoires serait-elle une donnée "définitive » indépendante de l'évolution historique, presque «naturelle », en raison des « origines communes»? Quand on parcourt le dictionnaire, on constate souvent une acception selon laquelle il y aurait des caractéristiques permanentes d'un ensemble de communautés en raison d'une culture d'origine faite nature. En d'autres termes, on constate une très nette sensibilité lusotropicaliste et, en dernière analyse, primordialiste.

Du reste, cette sensibilité a manifestement guidé globalement le choix des auteurs. Ceux-ci, fort nombreux (350) sont dits, je souligne, «représenter les huit pays et régions lusophones les plus importants (Galice, Goa, Macao, Casamance) » (p. 6). C'est là un étrange critère de sélection des spécialistes. Le contenu s'en ressent fortement: le dictionnaire n'est pas pensé comme un outil - certes de langue portugaise - scientifique international écrit par des auteurs de toutes nationalités en fonction de leurs compétences, mais comme un ouvrage écrit par des lusophones (et partisans de la lusophonie) pour des lusophones (et partisans de la lusophonie). Le résultat est que la bibliographie de la grande majorité des notices est, très exagérément, surtout de langue portugaise (mais peu brésilienne), ignorant des ouvrages fondamentaux de langue anglaise et, parfois, française ou allemande. Il va de soi que les auteurs devaient tous savoir le portugais: ils ne devaient pas pour autant être quasiment tous citoyens de pays de langue portugaise se fondant presque uniquement sur des travaux de langue portugaise. On comprend mieux ce parti pris quand on lit la véritable profession de foi en page 5 :

"É que uma união feita na base da geografia, do regime político ou da religião obriga a alinhamentos de diversos tipos, de carácter transitório, ao passo que a do uso de uma língua comum é permanente, não obriga a nada e tudo possibilita. Essa vantagem do critério da língua

* À propos de F. Cristovão (ed.), M.A. Amorim, M.L. Garcia Marques, S. Brites Moita, Dicionário temático da lusofonia, Lisbonne, Textos Editores, 2005, 976 p., ISBN : 972-47-2935-4. 
tornou-se mais forte e persuasivo logo que se verificou que nem a existência de uma língua oficial prejudicava as línguas nacionais de cada país, nem estas aquela... " [souligné par moi]

Ainsi la «langue commune » est une donnée neutre, permanente, elle «n'oblige à rien » et « rend tout possible »... En d'autres termes, voici à nouveau la langue faite patrie... Est ainsi totalement oubliée la régression des langues africaines, rejetées de la modernité par des systèmes scolaires pensés (presque) uniquement en langue portugaise, par et pour elle. La coopération portugaise qui - tout comme la française - est vertébrée par l'obsession de la diffusion de sa langue, n'est pas non plus questionnée.

Les entrées sont ordonnées classiquement par ordre alphabétique mais un organigramme les trie thématiquement (p. 7-18). Un tel dictionnaire ne se lit pas, il s'utilise en fonction des besoins de chacun. On regrette qu'il n'y ait pas un index général à la fin.

Pour le thème Antropologia, j’ai testé «Antropologia de Moçambique » (p. 47-51). Cet article de Ana Maria Loforte est un exposé fidèle de la vie mouvementée de cette discipline après l'indépendance. Il est dommage cependant que l'auteur ait commencé son propos à la lutte armée, sans aborder par conséquent l'anthropologie coloniale du Mozambique (qui inclut quand même quelques bons auteurs !). Inversement, elle ne cite pas la critique étrangère de la situation de l'anthropologie au Mozambique après l'indépendance (par exemple le célèbre article de G. Geffray, «Fragments d'un discours du pouvoir (1975-1985) : du bon usage d'une méconnaissance scientifique », Politique africaine, mars 1988, XXIX : 71-85).

\section{Rien n'a-t-il donc changé ?}

Dans le thème Conceitos especiais (sic, ne serait-ce pas plutôt especificos??), j'ai testé «Etnia », «Lusofonia » (ainsi que «Lusografia »), «Luso-tropicalismo » et «Multiculturalismo». On a là des entrées qui permettent de replacer la lusophonie dans un contexte plus vaste. L'entrée «Etnia » (Isabel Trindade, p. 300-302) souffre du défaut inverse de celui généralement constaté : c'est une trop brève mais stimulante présentation internationale de la problématique, mais qui n'étudie pas l'usage de ce concept dans les pays lusophones. L'entrée «Lusofonia » (de F. Cristovão, p. 652-656) est un exposé détaillé de la question, historique et sociolinguistique. L'auteur cependant ne discute pratiquement pas le statut social de la langue et se contente ainsi du nombre des habitants des pays comme fondant le nombre de lusophones (p. 656)! Des références bibliographiques non portugaises auraient facilement permis de nuancer cette approche. Cela est d'ailleurs fait dès l'entrée suivante, «Lusografia » (de J.-M. Massa, p. 656-657) : l'inventeur du concept de lusographie ne pouvait pas ne pas en expliciter la nuance!

L'entrée «Luso-tropicalismo» a pour auteur Adriano Moreira (p. 657-661). C'est évidemment une notice très importante pour ce dictionnaire, et on peut de ce fait questionner le choix d'un tel auteur, lusotropicaliste convaincu. Cela n'enlève pas l'intérêt du texte, qui inclut en peu de pages l'essentiel de la biographie intellectuelle de Gilberto Freyre. Mais on ne sait jamais bien ce qui est citation de G. Freyre ou point de vue de l'auteur. Ainsi le Brésil comme «équili- 
bre d'antagonismes » (équilibre entre la culture et l'économie, entre le maître et l'esclave, etc.) est une citation de Freyre (p. 658), mais reproduite avec empathie ; de même le rapport de l'Unesco de 1953 sur le concept de race est cité, pour le chapitre particulier qu'il consacre à l'action coloniale portugaise, comme si ce rapport était indépendant des courants idéologiques du début des années cinquante! Et Adriano Moreira souligne lui-même - ce n'est pas une citation de Freyre - que la différence entre les diverses colonisations tient en ce que " a amorosidade seja o valor dominante ou que a discriminação seja o príncipio estruturante » (p. 659)! Écrire un tel poncif, encore, en 2005! Pour autant, il remarque très justement que le lusotropicalisme, «modèle observé » par G. Freyre dans le Nord-Est brésilien (Recife) est devenu «modèle observant» pour le reste du Brésil et les autres territoires colonisés par le Portugal. Mais il n'y a aucune critique dans le texte sur la manière dont G. Freyre «observa » le lusocolonialisme (fait extension du lusotropicalisme pensé au Brésil) lors de son fameux voyage dans les colonies portugaises au début des années 1950. Pis, A. Moreira croit utile de noter que, alors que la quasitotalité de l'administration à Goa était peuplée de Goanais - «preuve », sans doute, de lusotropicalisme et d'absence de colonialisme, mais sans qu'il ne dise qu'il s'agissait, en majorité écrasante, exclusivement de Brahmanes de caste supérieure -, l'intervention indienne (1961) aurait été une «flagrante violation de la Charte de l'Onu et du Droit international» (p. 659). L'auteur considère que Goa aurait dû avoir son indépendance propre, comme si cette hypothèse avait été ne serait-ce qu'imaginée par Salazar ou demandée par des Goanais! Enfin dans la situation actuelle de «sociétés multi-ethniques et multiculturelles » qui remplaceraient progressivement les « uniformités ethnicoculturelles du modèle national » (p. 660), le lusotropicalisme reprendrait sa pertinence comme modèle "observé » et «observant». Il s'agit là de la version lusotropicaliste - fréquente - de la lusophonie conçue comme identité certes multi-ethnique et flexible mais néanmoins identité commune parce que issue de la colonisation portugaise. On est loin de la pensée critique d'un Eduardo Lourenço définissant la lusophonie comme « espace spécifique d'intersection avec d'autres identités » et non pas elle-même une identité métissée. Eduardo Lourenço n'est d'ailleurs pas cité dans la bibliographie finale de l'article. Cette dernière ignore absolument tout des travaux nombreux sur le lusotropicalisme élaborés en France (par exemple les 586 pages du vol. IV de Lusotopie, 1997, sur «Lusotropicalisme. Idéologie coloniale et identités nationales dans les mondes lusophones »), dans les pays anglo-saxons et bien sûr au Portugal (par exemple, Claudia Castelo, O modo português de estar non Mundo. O luso-tropicalismo e a ideologia colonial portuguesa (1933-1961), Porto, Afrontamento, 1999, 166 p.). La quasi-totalité des références sont, d'ailleurs, antérieures à $1970 \ldots$

En contraste, la notice, bien plus courte, sur le multiculturalisme (de António Custódio Gonçalves, p. 738-739) est un bref exposé, ouvert aux débats internationaux. On regrettera juste que le lien ne soit pas fait avec le postcolonialisme.

Dans le thème Direito, j'ai testé «Cidadania lusófona » (de Jorge M. Pereira da Silva, p. 166-167). L'auteur fait remarquer dès le départ que la «citoyenneté lusophone» ne pourra jamais être une citoyenneté au sens traditionnel et rigoureux du terme; en revanche, elle peut consigner un ensemble de droits dérivant des citoyennetés nationales. Le Cap-Vert a montré la voie, en adoptant unilatéralement, en 1997, un «statut du citoyen lusophone » qui accorde des droits 
amples aux citoyens des pays de la Communauté des pays de langue portugaise (CPLP).

Dans le thème Economia, les divers articles «Economia de Angola », «...do Brasil », «... de Guiné-Bissau », «... de Moçambique, «...de Portugal », «...de São Tomé e Príncipe », respectivement de Manuel Enes Ferreira (p. 229-241), Virgínio do Rosário Lopes (p. 241-252 et 252-256), José Manuel Mosca (p. 256266), Júlio Neves (p. 266-276) et à nouveau de V. do Rosário Lopes (p. 276-279), sont de bons états des lieux. On regrettera que ces diverses «fiches » n'aient pas suivi un plan plus uniforme, afin de permettre la comparaison. Il n'y a pas d'article sur l'économie de Timor, ni de la Galice, de Goa ou de Macao.

Dans le thème História, j’ai testé naturellement «Descolonização das colónias portuguesas » (p. 211-212, une seule page...). On peut, à nouveau, questionner le choix de l'auteur, António de Almeida Santos. Un acteur des événements est-il le mieux placé pour écrire dans un dictionnaire, en principe œuvre de chercheurs? La notice est loin de répondre aux besoins, d'autant que les fiches « História de Angola » (Isabel Castro Henriques \& Ana Martinho, p. 442-456), «...de Cabo Verde » (Ilídio Baleno, p. 462-470), «...de Goa» (Teotónio Ronald de Souza, p. 470-473), «... de Guinée-Bissau » (José da Silva Horta \& Eduardo Costa Dias, p. 473-483), «... de Moçambique » (João Paulo Borges Coleho \& Amélia Neves Souto, p. 483-493), bien plus consistantes, voire très détaillées et de bonne qualité, ne traitent pas de la décolonisation non plus (ce sont surtout des fiches d'histoire coloniale). Cependant la fiche de l'ancien ministre de la décolonisation n'est pas mauvaise, même si des jugements de valeur étonnants la parsèment (par exemple, si l'acharnement du salazarisme à ne pas décoloniser est qualifié d'« erreur majeure » - on est bien d'accord! -, c'est parce qu'au bout de treize années de guerre les dirigeants des mouvements de libération avaient «absorbé l’idéologie [des] pays de l'orbite soviétique »: une «erreur majeure », seulement à cause de cela ?).

\section{Introuvable " expression portugaise "}

Dans le thème Instituição - dans lequel bizarrement sont incluses les fondations et les associations -, j'ai testé «Fundação José Eduardo dos Santos » (p. 335), dont l'auteur est le secrétariat de rédaction du «Dictionnaire ». C'est une toute petite fiche qui ne nous apprend rien (résumé du programme officiel de cette fondation) alors pourtant qu'au moins une étude approfondie, de Christine Messiant, a été faite sur la Fesa comme exemple archétypique du néopatrimonialisme et de la corruption institutionnalisée en Angola («La Fondation Eduardo dos Santos (Fesa). À propos de l' "investissement" de la société civile par le pouvoir angolais ", Politique africaine, 73, 1999: 82-102). Cette étude fut d'ailleurs publiée en portugais par la presse angolaise d'opposition, ce qui valut à notre amie quatre années d'interdiction de séjour dans le pays...

Toujours dans le même thème, la petite fiche «Palop » (de F. Cristovão, p. 783) n'est pas satisfaisante parce qu'elle évite d'aborder la vraie question: si ces pays ont adopté un tel sigle, ce n'est nullement, comme le dit l'auteur, en succession de la Conférence des organisations nationalistes des Colonies portugaises (la CONCP, orthographiée ici «CONCOP»), mais justement parce que ces pays ne 
voulaient pas s'appeler «Pays africains d'expression portugaise » et encore moins «lusophones ». Ils ont adopté un sigle neutre, relatif à l'usage officiel de la langue portugaise - mais leur expression, quelle qu'en soit la langue, était africaine et non portugaise. Ils n'ont pas accepté non plus que la CPLP s'appelle «Communauté lusophone ». L'affirmation selon laquelle le sigle «Palop» serait de plus en plus remplacé par l'expression "Pays africains lusophones » est une assertion lisboète que je n'ai jamais constatée sur le terrain. Enfin, contrairement à ce qu'écrit l'auteur de la notice, les «Palop» n'ont jamais été une «institution ». Ce qui l'a été, sans existence permanente du reste, fut la tenue de "Cimeira dos Cinco ». Institution tellement lusophone qu'elle servait explicitement à empêcher des réunions communes avec le Portugal et le Brésil, dont la pratique ne commença qu'à la fin des années 1980.

Dans le thème Lingua, il y a des fiches sur les langues de chacun des pays, sauf le Brésil : «Línguas de Angola » (Irene Guerra Marques, p. 608-609), « ... de Cabo Verde » (Ernesto Pardal, p. 609-610), «...da Guiné-Bissau » (Luigo Scantarburlo, p. 610-611), «...de Macau » (M. José dos Reis Grosso \& Raul Legal Gaião, p. 611), «... de Moçambique » (une fiche importante, par Gregório Firmino, p. 611-614), «...de São Tomé e Príncipe » (Fernanda Pontífice, p. 614), «...de Timor» (Luís Costa, p. 614-615). Sur le Brésil, il y a une fiche sur les variations du portugais du Brésil, de Silvia Brandão \& Afrânio Gonçalves Barbosa (p. 855-856), mais rien sur les langues indiennes, ce qui est d'autant plus étonnant que les auteurs précisent très justement que «le portugais brésilien s'est constitué au cours d'un processus de réduction de la complexe configuration ethnico-linguistique engendré par le génocide patent de diverses nations et l'acculturation obscure de segments bilingues » (p. 856). Avec ces auteurs-là, au moins, on est loin du lusotropicalisme! $\hat{A}$ ces fiches, il faut ajouter celle sur les «Crioulos » (Dulce Pereira, p. 198-199) dont on regrette qu'elle soit si courte et, surtout, mentionne mais ne discute pas suffisamment la question de la pratique actuelle des créoles d'Asie et d'Amérique hors Brésil. Il est contestable, du reste, d'avoir une seule fiche sur "les créoles » (on aurait trouvé justifié qu'il y en ait au moins une pour chacune des grandes familles: créoles de Haute-Guinée, du Golfe de Guinée, indo-portugais, lusomalaio-indonésien, luso-chinois, luso-caribéen). La langue mirandaise, la seule minorité linguistique territorialisée - de surcroît officiellement reconnue, au Portugal depuis 1998 -, n'a pas sa fiche.

\section{La lusophonie, un fait de portugalité ?}

Faire un dictionnaire de ce type n'était pas facile. Mais ici, l'orientation générale évidemment non uniforme dans toutes les fiches - a été un obstacle. Tout d'abord, il y a une forte orientation littéraire, ce qui est déjà un choix d'approche de la lusophonie (par exemple il y a de nombreuses et intéressantes fiches sur des écrivains, mais que l'on ne retrouve pas pour d'autres personnages historiques ou scientifiques, etc., qui ont pourtant concouru au rayonnement des pays de langue portugaise, y compris de la langue portugaise elle-même). Cette orientation linguistico-littéraire n'a pas permis une approche de la lusophonie débarrassée de l'idéologie lusotropicaliste. Le concept de lusotopie (comme ensemble des espaces façonnés par l'histoire et la colonisation portugaises, quelle que soit la langue actuellement 
parlée), n'a pas de fiche dans le dictionnaire même un auteur, Teotónio R. de Souza l'utilise (p. 473) dans la fiche «História de Goa» (p. 470-473). Par là, il veut montrer que Goa est un excellent exemple de lusotopie non lusophone. Cette approche est une exception dans le dictionnaire.

L'approche par trop linguistique de la réalité des pays de la CPLP - en réalité une approche trop portugaise de ces pays - explique probablement certaines carences. S'il y a bien une fiche "Colonialismo " (p. 184-186) dont on est quand même stupéfait de constater qu'elle a été confiée à un ancien ministre des Colonies de A. de Oliveira Salazar, Adriano Moreira - le fait qu'il soit indéniablement un éminent intellectuel ne rend pas tout possible -, il n'y a pas de fiche " Colonização » et surtout, pas de fiche «Pós-colonialismo ». Des institutions internationales comme la CPLP ou l'Uccla (Union des villes capitales de langue portugaise) sont classées dans le chapitre Instituições (tout court) et non pas dans le chapitre Organizações e instituições internacionais : seraient-elles des instances à ce point «portugaises » qu'elles ne seraient pas - pas ressenties par les auteurs du «Dictionnaire» en tout cas comme internationales?

Le thème Movimentos sociais est exclusivement constitué de fiches sur les... maçonneries et les «Misericordias", à l'exception d'une très petite fiche sur les «Movimentos negros no Brasil» (Maria Aparecida Ribeiro, p. 737-738). N'y a-t-il pas de syndicalisme (pas de fiche) et d'associations internationales de syndicats lusophones (pas de fiche), de féminisme (pas de fiche), de mouvement estudiantin (pas de fiche), de luttes pour la terre (ne serait-ce qu'une fiche sur le Movimento dos Trabalhadores rurais sem terra au Brésil ? !) dans les pays de la CPLP ? La Révolution des (Eillets de 1974 (pas de fiche) n'a-t-elle pas été un formidable mouvement social qui a fait plus pour le rayonnement du Portugal et de sa langue que cinquante années de propagande salazariste?

La «portugalité » du dictionnaire est patente encore dans le thème Património: on y trouve rien moins que 33 fiches sur les divers "patrimónios portugueses » dans les pays d'Europe, d'Asie et d'ailleurs (p. 58, 175-177, 783-853), auxquelles il faut ajouter encore deux fiches sur la «diaspora» portugaise (João de Sousa Campos, p. 219-222), pour une seule fiche sur les nombreuses "migrations" angolaise, brésilienne, capverdienne, santoméenne, goanaise dans les pays les plus divers (Maria Beatriz da Rocha Trindade, p. 705-707): ou bien il fallait laisser à cette auteure un espace bien plus grand, ou alors faire autant de fiches que nécessaire. C'est dommage en particulier pour la puissante communauté des CapeVerdean Americans du Massachusetts, bien étudiée par l'université de Boston, dotée de ses radios, carnavals et journaux propres.

Significativement (car ils ne sont pas dans un «pays lusophone »?), on n'a pas de fiche en propre sur les Agoudas (descendants de trafiquants d'esclaves ou d'esclaves du Brésil revenus dans ce qui devint ensuite Bénin, Togo et sud-ouest nigérian). Référence y est faite dans la fiche "Património brasileiro no mundo não lusófono » (Alberto Costa e Silva, p. 783-786). Cette fiche est bien intéressante au demeurant: mais doit-on encore considérer les Agoudas d'Afrique occidentale comme des «Brésiliens»? Sont-ils seulement un «patrimoine » ? En revanche, on n'a (presque) rien sur les Burghers du Sri Lanka (une simple allusion dans la fiche " Croulos», p. 199). Sur les Kristang de Malaca (également « listés» dans la fiche «Crioulos »), il faut une fois de plus aller chercher dans les fiches sur les patrimoines 
portugais - ce qui est assez horripilant. Les Burghers et les Kristangs (et autres luso-créolités malaio-indonésiennes) ne sont pas plus des Portugais (ou même des «post-Portugais ») que les Angolais ou les Mozambicains, même s'ils sont, entre autres, le produit de l'histoire coloniale (fiches de Silvana Pires, p. 818-825, et de José Maria Cabral Ferreira, p. 832-834).

Du reste, le thème Migração e Comunidade est lui aussi trop peu " déportugalisé » : cinq des neuf fiches concernent exclusivement le Portugal. Il y en a une sur l'émigration bissau-guinéenne (Celestino Monteiro de Macedo, p. 284), qui s'ouvre sur l'affirmation que "As fontes [sobre] os emigrantes guineenses são raras, senão inexistentes", alors que de nombreux articles ont été publiés, en portugais, sur ce thème, ne serait-ce que par Eduardo Costa Dias, présent dans ce même Dictionnaire comme auteur d'autres fiches (par exemple celle, déjà citée, sur l'histoire de la GuinéeBissau)! Il n'y a pas de fiche en propre sur l'émigration capverdienne (il faut aller en chercher les données dans la notice «Geografia de Cabo Verde» (Isabel Marques Medeiros \& José Malheiros, p. 373-390) ou retourner à la trop courte fiche «Migrações no paises lusófonos » déjà citée (M.B. da Rocha-Trindade, p. 705-707), mais qui en reste au plan statistique et n'avait pas à aborder les questions patrimoniales ou sociales.

L'impression globale est donc fort mitigée. D’une part il est certain que cet épais dictionnaire sera parfois utile de par la quantité d'informations qu'il contient et la qualité de certaines notices. Mais d'autres sont bien plus contestables et c'est la conception d'ensemble qui est problématique. Elle ne rompt en rien avec la vision de la lusophonie comme simple dilatation de lusitanité, voire de portugalité, et semble, dans la plupart des notices, très éloignée des courants contemporains de la recherche internationale sur ces aires. Une fois de plus, on a l'impression que des Portugais ont voulu parler à des Portugais de la grandeur de leur nation, quand il était possible de réaliser un outil scientifique et encyclopédique d'excellence internationale, bientôt traduit en anglais, en français, etc.

Avril 2007, Michel Gahen

CNRS, Centre d'étude d'Afrique noire Université de Bordeaux, Sciences Po Bordeaux 


\section{LES MIGRANTS CARIBÉENS : RÉSEAUX ET DESCENDANCE Coordination :}

\section{Cédric Audebert}

\section{et Hervé Domenach}

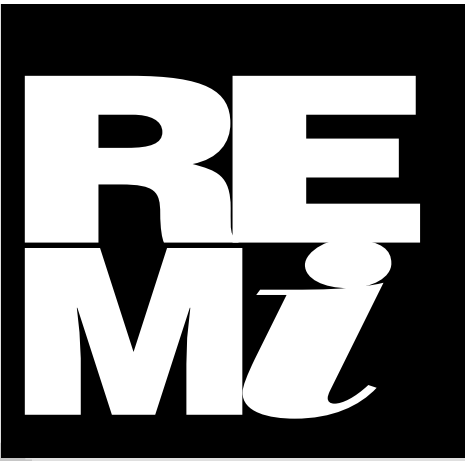

Revue Européenne des Migrations Internationales

\section{Éditorial}

Cédric Audebert et Hervé Domenach : Les migrants caribéens : réseaux et descendance

Irmary Reyes-Santos : Capital neoliberal, raza, migración: análisis comparativo de relaciones domínico-haitianas y domínico-puertorriqueñas

Stéphanie Condon et Margaret Byron : Migrations, résidence et représentations de la famille : les familles caribéennes en France et en Grande-Bretagne

Cédric Audebert : L'intégration des Antillais en France et aux États-Unis : contextes socio-institutionnels et processus de territorialisation

Margarita Mooney : Structures de médiation et intégration des immigrants haïtiens à Paris

Dwaine Plaza : Transnational Return Migration to the English-speaking Caribbean

Note de recherche

Terry-Ann Jones : L'intégration des immigrants jamaïcains à Miami et Toronto

Volume $24-N^{\circ} 1-2008$

ASSOCIATION POUR L'ÉTUDE DES MIGRATIONS INTERNATIONALES (AEMI)

MSHS - 99 Avenue du Recteur Pineau - 86000 POITIERS

remi@mshs.univ-poitiers.fr

http://remi.revues.org/

ISSN 0765-0752 - ISBN 978-2-911627-48-2 